humbly request that our article be read in that light.

We are not social scientists and so were unaware of the weight of that term in some circles (as, evidently, were the peer reviewers). Our intention was neither to draw any conclusion about the potential benefit of the spread of biotechnologies we studied nor to provide any comment on concepts of governance of these powerful technologies. The study of those phenomena and how to support them is indeed worthy, but beyond the scope of our original study.

We wonder, as we communicate our findings to a wide audience: what term of art should we use to describe the concept of not just the spread of biotechnologies, but the spread of the technologies to those with fewer resources and less training or education?

We appreciate the interest in our work and the call to all scientists to keep the broader implications of their work always in mind.
Shawn S. Jackson (D) ${ }^{凶}$, Louise E. Sumner and Rocco J. Casagrande

Gryphon Scientific, LLC, Takoma Park, MD, USA.

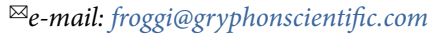

Published online: 26 March 2021

https://doi.org/10.1038/s41587-021-00892-w

\title{
Alternative hosts as the missing link for equitable therapeutic protein production
}

To the Editor - In your May 2020 issue, a Comment by Brian Kelley ${ }^{1}$ highlighted a proposed strategy for developing therapeutic monoclonal antibodies (mAbs) rapidly in the context of a pandemic by streamlining the existing manufacturing infrastructure in the biopharmaceutical industry. The author outlined how, by accepting defined risks in cell line development and encouraging proactive manufacturing processes (Fig. 1), the current 'best-in-class' timelines for transitioning a lead $\mathrm{mAb}$ candidate into a phase 1 clinical study could be reduced from 10-12 months to 5-6 months. We agree that reducing timelines to Investigational New Drug applications (INDs) is critical in pandemic settings like the one we face now with coronavirus disease caused by SARS-CoV-2, but manufacturing innovations that leverage alternative hosts for expression could enable even faster transitions and increased global access to therapeutics.

Recombinant therapeutic mAbs are being developed for the current pandemic, with over 40 candidates targeting SARS-CoV-2 announced and in the pipeline to the clinic $^{2,3}$. As $>100$ million people have contracted COVID-19 globally and existing systems for response are tested, we should carefully consider all opportunities to reduce the timelines for advancing therapeutic candidates to phase 1 studies and beyond. We suggest that the development timeline could be reduced to just $12-15$ weeks (Fig. 1), or by another factor of 2 , using alternative non-mammalian hosts to produce mAbs. We further highlight additional developments needed to realize such accelerated timelines routinely by further diversifying the global manufacturing infrastructure for potential increased access for patients globally. In a pandemic, every week counts for all patients.

\section{Alternative hosts reduce key technical risks in accelerated development} The COVID-19 pandemic has highlighted the importance of timely, parallel transitions for multiple lead candidates to first-in-human studies (phase 1)

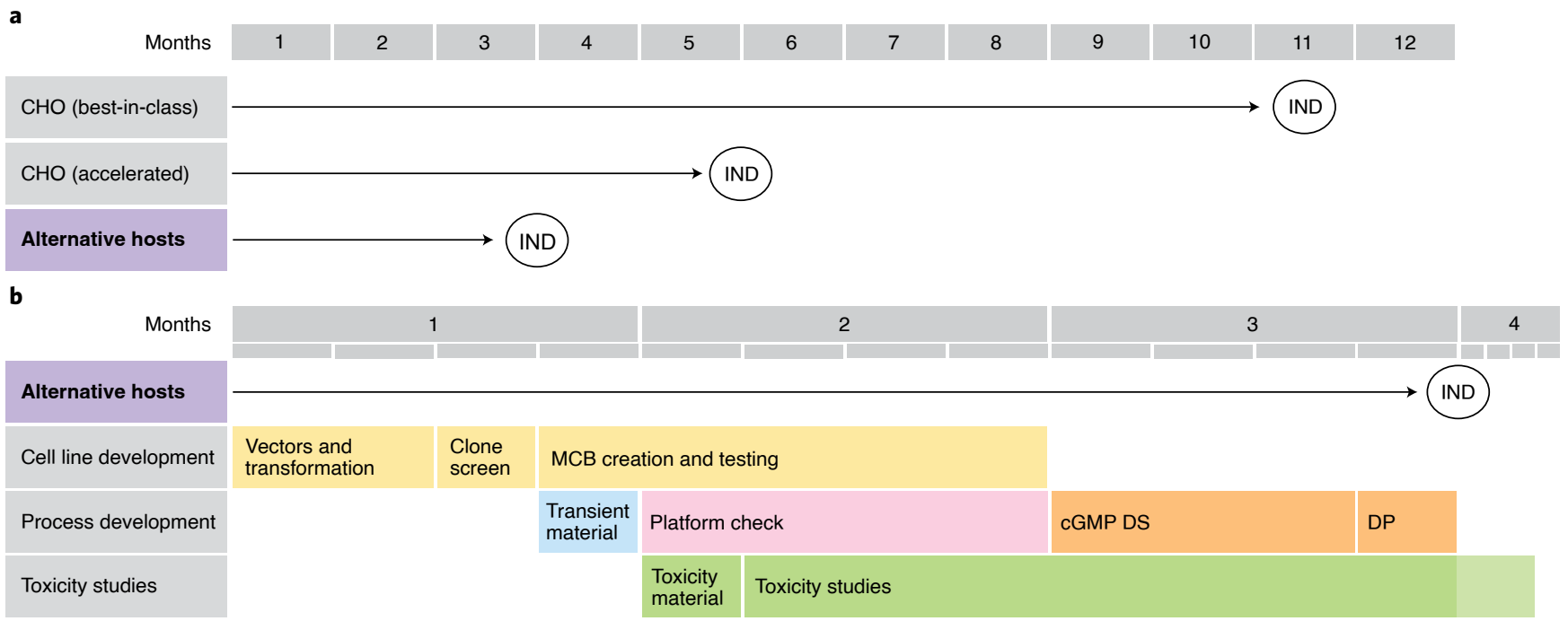

Fig. 1 Accelerated timeline to phase 1 IND using alternative hosts. a, Comparison of the best-in-class and accelerated development timelines using Chinese hamster ovary $(\mathrm{CHO})$ cells as presented by Kelley to a potential timeline using alternative hosts. $\mathbf{b}$, Accelerated activities on the critical path to phase 1 IND for alternative hosts. 'Platform check' refers to minimal development of necessary process, formulation and analytics. MCB, master cell bank; DS, drug substance; DP, drug product. 
and beyond. The success rate for biopharmaceuticals is generally $20 \%$ (ref. ${ }^{4}$ ) and may be even lower when treating a novel, minimally characterized pathogen. To compress the timeline to current good manufacturing practice (cGMP) production of an IND-enabling lot, Kelley ${ }^{1}$ suggested modifying the steps required for creation and preparation of the master cell banks (MCBs). These modifications increase technical risks, but are aimed at minimizing the number of days and iterations for cultivation to generate final clones and cell banks.

Alternative hosts can reduce or eliminate the above added technical risks. We define alternative hosts as cell lines or microorganisms used for protein expression not used routinely at present in the manufacturing of commercial recombinant biopharmaceuticals marketed in the United States or the European Union. Examples of such alternative hosts include yeasts (Komagataella phaffii (known formerly as Pichia pastoris), Hansenula polymorpha or Kluyveromyces lactis), filamentous fungi (Trichoderma reesei, Aspergillus oryzae or Myceliophthora thermophila) and protozoa (Leishmania tarentolae or Tetrahymena thermophila). Depending on the class, these hosts have one or more intrinsic features that reduce the technological risks presented by compressed timelines for development and manufacturing. These features include fast growth rates, invulnerability to adventitious agents, reduced numbers of secreted host cell proteins, compatibility with low-cost processing steps, and robustness against harsh process conditions, among others ${ }^{5,6}$.

Extensive screening of hundreds to thousands of transfected clones of $\mathrm{CHO}$ cells is common in the identification of optimal MCB lines to account for their clonal variation and inherent genetic instability. ${ }^{7}$ Methods for targeted integration of genetic constructs may reduce the number of clones screened to avoid suboptimal lines, but this technical risk can be minimized further for many alternative hosts. Yeasts, for example, have a remarkably stable genome and genes are integrated precisely when creating clones $^{8}$; as such, clones are engineered, not selected. In our experience, the variation in productivity or quality among five to ten selected clones for a given expressed protein is minimal. The implication of this deterministic editing is that initial strains are created for purpose and are ready to transition to master cell banking within 2-3 weeks of receiving DNA. This direct selection of the intended clone also minimizes the potential risks of variation in quality attributes that may arise when using pools of transfectants to generate first quantities of a drug substance before changing to a final selected clone for later stages in production.

Some alternative hosts can also further compress the time required for key activities in cGMP production to enable an IND. These include the establishment of the $\mathrm{MCB}$, the cultivation of cells to inoculate a production-scale bioreactor, and the production cycle itself. The growth rate of the cells themselves sets the cadence of these activities. CHO cells double every 14-36 hours depending on the stage of growth and conditions ${ }^{5}$. Alternative hosts such as yeast, filamentous fungi and protozoa grow nearly tenfold faster than $\mathrm{CHO}$ cells, with doubling rates typically ranging from 1.5 to 4 hours ${ }^{5,9}$. This inherent difference makes it possible to grow the volumes of cells needed to establish a MCB four- to sixfold faster than for $\mathrm{CHO}$ cells. Furthermore, generating the volume of $\mathrm{CHO}$ cells needed for the seed train and production at $\sim 2,000$ liters requires $\sim 23$ doubling times, or about 22 days. The accumulation of comparable biomass using an alternative host requires only $\sim 3$ days with an assumed doubling time of $3 \mathrm{~h}$. These steps are on the critical timeline for any new candidate, and thus gains here can reduce the time to the release of an IND-ready lot of the candidate.

In a pandemic resulting from a novel pathogen, maximizing the number of candidates transitioning to phase 1 clinical studies will depend on the most efficient production cycles available within existing facilities. Failed or missed production cycles present a tangible opportunity cost to society when other candidate therapeutics and vaccines await the same fixed manufacturing capacity. Alternative hosts can offer an advantage here as well. The typical fermentation time for yeasts or filamentous fungi in fed-batch operations is 7-10 days, compared with 14-21 days for $\mathrm{CHO}$ cells. Assuming similar efficiencies in a protein-A-based process for recovery, this difference in time means that an alternative host can achieve a comparable volumetric productivity of a mAb with $\sim 50 \%$ of the titer of a CHO-based process. Titers of $\sim 1.5 \mathrm{~g} \mathrm{~L}^{-1}$ have been demonstrated with $K$. phaffii, and up to $20 \mathrm{~g} \mathrm{~L}^{-1}$ reported for certain fungi; even though these processes are still underdeveloped compared with CHO-based protocols ${ }^{10,11}$, these titers compare favorably with those observed for $\mathrm{mAbs}$ produced in $\mathrm{CHO}$ cells, from early stages of transient expression and current commercial processes $\left(1-5 \mathrm{~g} \mathrm{~L}^{-1}\right)$ to highly intensified processes achieved with focused development $\left(10-15 \mathrm{~g} \mathrm{~L}^{-1}\right)$. Thus, it is feasible to achieve comparable production with less total time in a facility.
When combined, the gains possible from clonal selection through production with an alternative host could reduce the operational timeline from sequence to IND to only 12-15 weeks (Fig. 1). Consequently, diversification of global manufacturing of mAbs to include ones produced with alternative hosts could better minimize risks by both reducing timelines and further distributing risk for timely clinical transitions of lead candidates.

\section{Alternative hosts can produce safe and efficacious products}

In the Comment, Kelley dismissed the utility of alternative hosts in a pandemic response, citing potential unknown risks associated with non-mammalian-derived recombinant proteins, such as "rare mAb post-translational modifications," including "unusual glycans" and "host cell proteins" ${ }^{1}$. This perspective ignores both the extensive clinical experience with products produced in alternative hosts globally and the risk-based approach now routinely used in development to temper such concerns ${ }^{12}$. Many licensed parenteral biopharmaceuticals are produced globally in alternative hosts today and safely administered to millions of patients every year, including biosimilars and originator molecules, such as insulin and enzyme replacement therapies ${ }^{13,14}$.

Focusing on mAbs, three examples illustrate that high-quality therapeutic mAbs with appropriate critical quality attributes can be achieved in an alternative host. In 2020, the US Food and Drug Administration (FDA) approved eptinezumab, a humanized aglycosylated immunoglobulin G1 (IgG1) $\mathrm{mAb}$ targeting the calcitonin gene-related peptide for treating chronic migraines ${ }^{15}$; in 2016, Bristol Myers Squibb published a phase 2 b study of clazakizumab, an anti-interleukin (IL)-6 IgG1 mAb for psoriatic arthritis with a safety profile consistent with that of other anti-IL-6 mAb therapies ${ }^{16}$; and in 2011, Merck published data on an anti-human epidermal growth factor receptor 2 (HER2) IgG1 $\mathrm{mAb}$, produced using a glycoengineered alternative expression system, that showed comparable pharmacokinetic profiles and activity to those of trastuzumab in a preclinical study, despite having glycan profiles differing from $\mathrm{CHO}$-produced material ${ }^{17}$. In all three of these examples, an alternative production host-the yeast $K$. phaffii-was used to successfully produce a therapeutic $\mathrm{mAb}$.

Furthermore, for any new product, analytical data on the candidate protein inform the determination of critical quality attributes (CQAs), regardless of the 
host. These data in turn inform a holistic control strategy to minimize residual risks to patients. Advanced analytical methods, such as mass spectrometry, and emerging multi-attribute methods are now common for characterizing recombinant biopharmaceutical products deeply and informing risk-based assessments of the potential clinical relevance of detected variants. The effects of glycan structures and compositions on safety and efficacy are well studied and can inform risk assessments for alternative hosts ${ }^{18}$. Of note, there are many aglycosylated antibodies or non-glycosylated antibody-like proteins (for example, nanobodies) that could be candidates for production in alternative hosts $^{19}$. Establishing an appropriate control strategy in accordance with current industry guidance and governing regulationsincluding end-product release testing for CQAs - is agnostic to the choice of host and is essential to assure safety for patients.

Alternative hosts may reduce other safety risks common to mammalian cells, such as adventitious contamination from viruses. This advantage has allowed minimal or no testing for virus in commercial products produced in yeast ${ }^{20}$. Such reduced testing requirements could both eliminate the need for viral testing in MCBs, as well as release testing, and reduce safety risks in accelerated development as compared with that required for mammalian cells.

\section{Readying tomorrow's agile manufacturing solutions}

Alternative hosts offer several potential benefits for speeding new candidates to the clinic and for maximizing the use of a manufacturing facility, during a pandemic or otherwise. In the current response to the COVID-19 pandemic, however, alternative hosts are primarily enabling only the preparation of recombinant vaccine candidates; they are not being used in $\mathrm{mAb}$ production. Certainly, the physical infrastructure and human capital for manufacturing mAbs with $\mathrm{CHO}$ cells is long established, and despite the extended timelines and finicky requirements of such cells, mammalian systems remain the option of choice.

Industry intransigence concerning alternatives to $\mathrm{CHO}$ cell manufacture may ultimately be shortsighted, however. We suggest that alternative systems can complement $\mathrm{CHO}$ cell manufacture, enabling rapid, robust, scalable and potentially more accessible solutions in future pandemics. To achieve this outcome, we suggest three critical requirements for routine manufacturing with alternative hosts: (i) establishment of a widely accessible collection of one or more suitable hosts with related tools for genome engineering, (ii) purposeful development of intensified processes to support efficient production and recovery, and (iii) acquisition of suitable equipment and facilities to support optimal use of such processes. Together, these advances would not only provide an agile infrastructure for pandemic response but also could serve to diversify the global manufacturing infrastructure and expand global access to medicines.

Rapid development, in a pandemic or otherwise, requires established components for cell line development to prepare production-ready strains. For $\mathrm{CHO}$ cells, these elements exist in the form of a variety of banked or proprietary cell lines and vectors for expressing heterologous genes. The present ecosystem, technical knowledge and trained workforces associated with $\mathrm{CHO}$-based production are the result of $>25$ years of industrial and academic advancements on a global scale. In contrast, most promising alternative hosts have been held under trade secret or patents or simply dismissed as irrelevant to pharmaceutical-grade manufacture. Companies protecting these technologies (for example, Tetragenetics, Dyadic, GlycoFi/Merck, Pfenex and the Jena, Germany-based biotech Jena Bioscience) have largely relied on business models that constrain access to the host through restrictive or expensive licensing agreements or that use them exclusively for internal purposes. These business models may provide benefit to a limited set of investors and shareholders, but they have failed to maximize the potential impact of these new hosts for manufacturing and, ultimately, for patients and society.

Alternative models for developing hosts could accelerate their readiness, accessibility, and acceptance for future manufacturing solutions. Development of open-source strains and tools through joint academic and industrial partnerships, such as the AltHost Consortium, could accelerate the readiness of alternative hosts for future manufacturing capabilities focused on speed, flexibility and cost. An extra benefit of such open models is the ability to develop a common understanding of the relationships between product CQAs and host biology, as is well established for CHO-based products. Such knowledge can reduce both real and perceived risks such as those stated by Kelley and increase the risk tolerance of businesses when electing for speed in an emergent situation.

Traditional microbial fermentation has relied on technologies and media developed for intracellular expression and recovery. The advantages of sustained expression and secreted products offered by $\mathrm{CHO}$ cells in perfusion-based cultures should translate to eukaryotic microorganisms as well, though such approaches are largely underdeveloped. Extensive medium development and operational tuning has allowed sustained productivities of $1-2 \mathrm{~g} \mathrm{~L}^{-1} \mathrm{~d}^{-1}$ for $\mathrm{CHO}$ cells, and similar approaches could provide similarly large gains in productivity with alternative hosts ${ }^{21}$. Additionally, many eukaryotic microorganisms offer a reduced profile of host cell proteins, which should allow further reduction in the number of steps in recovery and buffers required ${ }^{5}$. Translating the learnings from process intensification and 'platform' processes for CHO-based production to new hosts will be crucial to achieving an efficient and diversified infrastructure for manufacturing.

Finally, for a timely transfer of manufacturing processes in a pandemic situation, it would be beneficial to further refine the manufacturing infrastructure itself for production with alternative hosts. The CHO-based manufacturing ecosystem relies on modular structural designs and prefabricated elements for facilities and largely standardized equipment-especially single-use systems-across the industry, from government-supported facilities to contract manufacturing organizations to biopharmaceutical companies ${ }^{22}$. Although not identical, these common architectures reduce complications in technology transfer from development to production.

The global capacity for production in microbial hosts is not all that far behind that of mammalian systems ( $\sim 5$ million liters compared with $\sim 10$ million liters for all mammalian hosts combined $)^{22}$, but there is limited comparability of constructed designs and installed equipment among these highly customized facilities. Variations among sites can limit the rapid transition of processes if needed to expand manufacturing capacity for a given product. Furthermore, there are limited options available for single-use systems in microbial cultivation presently; such equipment is important for the timely transition in a facility from one production process to another.

Alternative hosts are well-suited to merge with other innovations in manufacturing technologies such as single-use systems and continuous processing. Holistic integrated design of the equipment and facilities could enable entirely new models for cGMP production that are modular and truly multiproduct by design. We recently have demonstrated such a system for yeast that enables integrated, end-to-end manufacturing in a small-scale system ${ }^{23}$. Such technologies could be especially useful in a pandemic setting, where additional 
capacity must be quickly allocated for the rapid, early-stage development of many candidate therapeutics and vaccines. Standardized, multipurpose production systems could also provide more robustness in technology transfer of a candidate to multiple manufacturing sites in a pandemic. In total, advancing alternative hosts, innovative intensified processes, and supporting systems for manufacturing could augment existing infrastructure for mammalian-based manufacturing and further diversify global risks for constrained manufacturing capacity in emergent situations ${ }^{24,25}$.

\section{Making tomorrow now}

Manufacturing in the biopharmaceutical industry today already benefits many patients. The CHO-based infrastructure is being leveraged effectively to advance multiple mAbs as therapeutic candidates in the COVID-19 pandemic ${ }^{3}$. Nonetheless, a next-generation manufacturing solution that depends on fast and purpose-built alternative hosts with established baseline production processes and modular facilities could further enhance the speed of development and broaden the number of first-in-human studies to diversify risks in rapidly identifying successful treatments for a novel disease. Standardized and fit-for-purpose alternative hosts and production processes could also provide a common bridge across all operations within biopharmaceutical drug development generally, accelerating other candidate drugs from discovery and $\mathrm{R} \& \mathrm{D}$ to commercial production in less urgent times. One consequence of accelerating manufacturing timelines with alternative hosts is that other activities in drug development may become limiting, such as animal-based toxicity studies or analysis of product stability.

Beyond the opportunity to accelerate timelines in manufacturing for pandemic responses, alternative hosts could also contribute to equitable access to $\mathrm{mAb}$-based therapies. Currently, manufacturing mAbs using $\mathrm{CHO}$ cells, even in a pandemic, costs approximately $\$ 50-100$ per gram $^{26}$. The availability of $\mathrm{mAbs}$ in low- and middle-income countries remains low due to the costs of these products to public health systems $^{27}$. How to ensure equitable global access to successful $\mathrm{mAb}$-based therapeutics for COVID-19 remains, therefore, an open societal question. Modular, regional manufacturing solutions based on robust, transferable production processes and hosts with reduced or no cold-chain requirements (for example, lyophilized yeast) could offer better timely access in pandemics and in other times. Decentralized manufacturing can help reduce costs for available medicines ${ }^{28}$ and further innovations in the manufacturing systems used could further enhance affordable access.

The obvious arguments against further consideration for alternative hosts, or innovating new manufacturing of mAbs, are often simply that the industry today is not incentivized to do so with current prices in high-income countries or that the apparent business or regulatory risks are too uncertain. These arguments may be true if we do not also consider the recurring, offsetting societal costs of limited access to these products ${ }^{27}$. No transformation in the timeliness of development for pandemics or equitable access to products by patients will exist without intentional financing and substantial engagement by all stakeholders who would share in the outcomes. Increased and deliberate funding of partnered, open innovation by governments, foundations and industry, along with widespread diverse participation from academia to national labs to for-profit entities, will be needed to realize global readiness for new pandemics and transform health care for patients everywhere.

\section{Joseph R. Brady (D) 1,2 and \\ J. Christopher Love (D) 1,2凶}

${ }^{1}$ Koch Institute for Integrative Cancer Research, Massachusetts Institute of Technology, Cambridge, MA, USA. ${ }^{2}$ Department of Chemical Engineering, Massachusetts Institute of Technology, Cambridge, MA, USA.

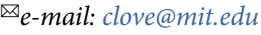

Published online: 29 March 2021

https://doi.org/10.1038/s41587-021-00884-w

References

1. Kelley, B. Nat. Biotechnol. 38, 540-545 (2020).

2. Hodgson, J. Nat. Biotechnol. https://doi.org/10.1038/d41587-02000005-z (2020)

3. FDA. Coronavirus Treatment Acceleration Program (CTAP). https://www.fda.gov/drugs/coronavirus-covid-19-drugs/ coronavirus-treatment-acceleration-program-ctap (2020).

4. Kaplon, H. \& Reichert, J. M. MAbs 11, 219-238 (2019).

5. Matthews, C. B. et al. Biotechnol. Bioeng. 114, 2432-2444 (2017).

6. Jiang, H. et al. Biotechnol. Bioeng. 116, 1449-1462 (2019).

7. Lewis, N. E. et al. Nat. Biotechnol. 31, 759-765 (2013).

8. Brady, J. R. et al. Biotechnol. Bioeng. 117, 543-555 (2020).
9. Fernández, F. J. \& Vega, M. C. Curr. Opin. Struct. Biol. 23, 365-373 (2013)

10. Potgieter, T. I. et al. J. Biotechnol. 139, 318-325 (2009).

11. Dyadic International. C1 expression system. https://www.dyadic. $\mathrm{com} / \mathrm{cl}$-technology/c1-expression-system/ (accessed 3 March 2021).

12. International Conference on Harmonisation of Technical Requirements for Registration of Pharmaceuticals for Human Use. ICH Harmonised Tripartite Guideline: Pharmaceutical Development Q8(R2) Step 4 version (2009); https://database.ich. org/sites/default/files/Q8 R2 Guideline.pdf

13. EMA. EMA European Public Assessment Report (EPAR): Semglee (Insulin Glargine, Biosimilar to Lantus) (EMA, 2018); https://www.ema.europa.eu/en/documents/assessment-report/ semglee-epar-public-assessment-report_en.pdf

14. Ghosh, P. K. J. Pharm. Pharm. Sci. 19, 552-596 (2017).

15. Dhillon, S. Drugs 80, 733-739 (2020).

16. Mease, P. J. et al. Arthritis Rheumatol. 68, 2163-2173 (2016).

17. Zhang, N. et al. MAbs 3, 289-298 (2011).

18. Reusch, D. \& Tejada, M. L. Glycobiology 25, 1325-1334 (2015).

19. Beck, A., Wagner-Rousset, E., Ayoub, D., Van Dorsselaer, A. \& Sanglier-Cianférani, S. Anal. Chem. 85, 715-736 (2013).

20. EMA. Jetrea Assessment Report (EMA, 2013); https:// www.ema.europa.eu/en/documents/assessment-report/ jetrea-epar-public-assessment-report en.pdf

21. Matthews, C. B., Kuo, A., Love, K. R. \& Love, J. C. Biotechnol. Bioeng. 115, 103-113 (2018).

22. Rader, R. A. \& Langer, E. S. Single-use technologies in biopharmaceutical manufacturing. in Single-Use Technology in Biopharmaceutical Manufacture 2nd edn (eds. Eibl, R. \& Eibl, D.) 193-200 (Wiley, 2019); https://doi.org/10.1002/ 9781119477891.ch15

23. Crowell, L. E. et al. Nat. Biotechnol. 36, 988-995 (2018),

24. Sharma, I., Wosińska, M., Kroetsch, A., Sullivan, H. \& McClellan, M. Ensuring sufficient manufacturing capacity for COVID-19 therapeutics. (Duke Margolic Center for Health Care Policy, 2020); https://healthpolicy.duke.edu/events/ webinar-ensuring-sufficient-manufacturing-capacity-covid-19. therapeutics

25. Langer, E. S. Genet. Eng. Biotechnol. News 36, 27-29 (2016). 26. Kelley, B. MAbs 1, 443-452 (2009).

27. Wellcome \& IAVI. Expanding Access to Monoclonal Products: A Global Call to Action (IAVI, 2020); https://www.iavi.org/ news-resources/expanding-access-to-monoclonal-antibodybased-products-a-global-call-to-action

28. Rathore, A. S. \& Shereef, F. Nat. Biotechnol. 37 498-501 (2019)

\section{Acknowledgements}

The authors thank K. Routenberg Love for comments and discussion. The content is solely the responsibility of the authors and does not necessarily represent the official views of the AltHost Consortium or the Bill and Melinda Gates Foundation, which support research in the lab of J.C.L.

\section{Competing interests}

J.C.L. receives sponsored research support at MIT from Amgen, the Bill \& Melinda Gates Foundation, Biogen, Genentech/Roche, Pfizer and Sanofi (collectively members of the AltHost Consortium). J.C.L. has interests in Amgen, Pfizer and Sunflower Therapeutics PBC. J.C.L. is an inventor on patents filed by MIT related to technologies described in this manuscript ${ }^{23}$. J.C.L.'s interests are reviewed and managed according to policies for potential conflicts of interest at MIT.

\section{Additional information}

Peer review information Nature Biotechnology thanks Justin Scheer and the other, anonymous, reviewer(s) for their contribution to the peer review of this work. 